\title{
¿POR QUÉ MOTIVOS SE ESFUERZAN LOS ESTUDIANTES EN EDUGACIÓN PRIMARIA?: UN ESTUDIO DE LA ORIENTAGIÓN MOTIVACIONAL POR GURSO Y SEXO
}

\section{FOR WHAT REASONS DO STUDENTS STRIVE IN PRIMARY EDUGATION?: A STUDY OF MOTIVATIONAL ORIENTATION BY COURSE AND SEX}

\author{
HÉCTOR GALINDO-DOMÍNGUEZ ${ }^{1}$ Y \\ MAITANE PEGAlajAR-MÉNDEZ ${ }^{2}$
}

Cómo referenciar este artículo/How to reference this article:

Galindo-Domínguez, H. y Pegalajar-Méndez, M. (2020). ¿Por qué motivos se esfuerzan los estudiantes en educación primaria?: Un estudio de la orientación motivacional por curso y sexo [For what reasons do students strive in primary education?: A study of motivational orientation by course and sex]. Acción Psicológica, 17(2), 123-134. https://doi.org/10.5944/ap.17.2.26044

\section{Resumen}

En el presente estudio se ha tratado de conocer cuáles son los motivos que tienen los estudiantes para esforzarse en el proceso de aprendizaje a través de la teoría de metas académicas en función del curso y el sexo. En esta línea, tomaron parte 1059 estudiantes (539 chicas y 520 chicos) de $4^{\circ}, 5^{\circ}$ o $6^{\circ}$ de Educación Primaria, quienes rellenaron el Cuestionario de Metas Académicas (García et al., 1998). Los resultados apuntan a que existía una correlación
\end{abstract}

significativa de intensidad leve-moderada entre las diferentes metas. De igual modo, los chicos presentaron puntuaciones significativamente superiores a las chicas en las metas de valoración social, y las chicas significativamente mayores a los chicos en las metas de aprendizaje. Respecto al curso, también se apreció como tanto las metas de aprendizaje como las metas de valoración social disminuían con el paso de los cursos. Estos resultados concuerdan casi en su totalidad con investigaciones desarrolladas en otro tipo de población.

Correspondence address [Dirección para correspondencia]: Héctor Galindo-Domínguez, Universidad de Deusto, España.

Email: hector.galindo@opendeusto.es

ORCID: Héctor Galindo-Domínguez (https://orcid.org/0000-0003-0562-160X).

${ }^{1}$ Universidad de Deusto, España.

${ }^{2}$ University of the Basque Country, España

Recibido: 30 de noviembre de 2019.

Aceptado: 28 de julio de 2020. 
Palabras clave: Educación Primaria; Orientación motivacional; metas de aprendizaje; metas de valoración social: metas de rendimiento.

\begin{abstract}
The purpose of the current research study has been to know which are the reasons students have to strive in the learning process through the goal theory, analysing course and sex differences. In this line, 1059 students (539 girls and 520 boys) of 4th, 5th and 6th grade of Primary Education took part filling out the Academic Goals Questionnaire (García et al., 1998). The results suggest that there was a significant correlation of mild-moderate intensity between the different goals. Likewise, the boys showed significantly higher scores than girls in social assessment goals, and the girls significantly higher that the boys in learning goals. With regard to the course, it was also noticed that learning goals and social assessment goals decreased with the passage of time. These findings agree almost entirely with investigations developed in another type of population.
\end{abstract}

Keywords: Primary Education; Motivational orientation; learning goals; social goals; achievement goals.

\section{Introducción}

Uno de los interrogantes, tal vez más importantes, que se ha planteado en el ámbito de la psicología escolar es el de dar a conocer los motivos por los que nos interesa aprender y desarrollar nuevos conocimientos y habilidades. En cualquier entorno educativo es medianamente fácil percatarse de que no todo el alumnado está interesado en aprender por los mismos motivos; o en otras palabras, no todos trabajan para alcanzar las mismas metas (García et al., 1998).

Para tratar de dar respuesta a por qué los y las estudiantes se aproximan y se involucran en una situación académica específica comenzó a desarrollarse una de las teorías más importantes en el estudio de la motivación escolar: la Teoría de Orientación a la Meta (Patrick et al., 2001).
La orientación a la meta podría considerarse como un modelo integrado de creencias, atribuciones y sentimientos que dirige las intenciones conductuales (González et al., 1996) y a su vez constituido por diversos modos de aproximación, compromiso y respuesta a las actividades que se plantean (Ames, 1992; González et al., 1996).

Dentro de los grupos de investigadores han sido a lo largo de los años varios los modelos propuestos para esta teoría (Matos y Lens, 2006), principalmente, difiriendo una de otra en la definición de metas, el nombre de cada constructo y en el número de metas propuestas (Linnerbrink y Pintrich, 2000). No obstante, la mayor parte de investigaciones sustenta la idea de la existencia de dos grandes tipos de metas: Las metas orientadas al aprendizaje o al dominio, y las metas orientadas al rendimiento (García et al., 1998; Ross et al., 2002).

Por una parte, cuando las y los estudiantes persiguen metas de aprendizaje, estos están preocupados por incrementar la comprensión del material, por enriquecer su conocimiento y por desarrollar nuevas habilidades a través del esfuerzo personal (Covington, 2000; Matos y Lens, 2006; Pintrich, 2000; Ross et al., 2005), por lo que hablamos de una meta puramente intrínseca y personal que el estudiante tiene de la tarea (Maehr y Midgley, 1996; Matos y Lens, 2006) cuya evaluación se basa en los estándares propios del estudiante (Matos y Lens, 2006). Este tipo de estudiantes, ante las adversidades son más propensos a buscar ayuda o a persistir en el esfuerzo, a través de la autorregulación, impulsados por la creencia de que el esfuerzo valdrá la pena con el paso del tiempo (Ames, 1992; Brophy, 2004; Dweck y Legget, 1998; Kohler y Reyes, 2010; Pintrich y De Groot, 1990).

Por otra parte, cuando el alumnado persigue metas de rendimiento, buscan obtener sentencias favorables de los demás así como evitar juicios negativos de la tarea realizada (Covington, 2000; Maehr y Midgley, 1996; Matos y Lens, 2006). Este grupo se caracteriza por mostrar un desempeño mayor al resto y evidenciar de esta manera su capacidad, obteniendo el reconocimiento público de su tarea (Maehr y Midgley, 1996; Matos y Lens, 2006; Ross et al., 2002). Este tipo de estudiantes, al tratar de obtener una recompensa deseada, no se centra tanto en buscar y desarrollar nuevas estrategias de aprendizaje sino que tien- 
den más a buscar estrategias que les aseguren un resultado positivo concreto (e.g., aprobar el examen) con el menor esfuerzo posible (García et al., 1998). Es de destacar que frecuentemente, los y las estudiantes motivados por este tipo de metas prefieren recibir un juicio favorable sobre una tarea relativamente fácil a correr el riesgo de obtener un juicio desfavorable ante una tarea más compleja y significativa (Miller et al., 1993).

Tal y como comentan Kohler y Reyes (2010), aunque la mayoría de modelos de orientación a la meta hayan propuesto, de forma general, los dos tipos ya descritos, investigaciones más recientes sustentan la idea de dividir las metas de rendimiento en dos, proponiendo por lo tanto un modelo de orientación a la meta trifactorial. En este sentido, en una serie de estudios (e.g., Elliot y Harackiewicz, 1996; Long et al., 2007; Middleton y Midgley, 1997; Pintrich y Schunk, 2002, entre otros) se comenzó a distinguir la orientación de metas de rendimiento, en dos nuevas metas: las metas de aproximación al rendimiento y las metas de evitación del rendimiento. Mientras que los estudiantes que se orientan a metas de aproximación al rendimiento se sienten motivados por superar a los demás y buscan sentencias favorables de competencia, los estudiantes que se orientan más hacia las metas de evitación al rendimiento prefieren evitar juicios desfavorables de competencia y por ende, podrían evitar las tareas de aprendizaje (Kohler y Reyes, 2010).

En los inicios de esta teoría, se sugería que las metas de aprendizaje y las metas de rendimiento eran excluyentes una de la otra y que además, generaban efectos opuestos sobre el aprendizaje (Kohler y Reyes, 2010). Sin embargo, la idea más actual es la de pensar que las metas de rendimiento pueden ser capaces de generar efectos positivos en las metas de aprendizaje (González et al., 1996; Harackiewicz et al., 1998; Ross et al., 2002).

Todos los tipos de metas comentadas hasta el momento están presentes de algún u otro modo en todo el alumnado haciendo que lo que varíe sea la intensidad con la que cada meta influye en lo académico (Heyman y Dweck, 1992; Meece, 1994; Seifert, 1995). Esta conclusión ha sido el resultado de realizar diversos análisis de correlaciones que constataron la existencia de una correlación positiva entre las metas de aprendizaje y metas de rendimiento (Ross et al., 2002), aunque su fuerza de correlación oscile entre baja a moderada (Middleton y Midgley, 1997).

En referencia al paso de los años, diversos estudios citados en Ross et al. (2002) sostienen la idea de que los y las estudiantes de menor edad tienden a adoptar metas de aprendizaje con mayor intensidad que los estudiantes de mayor edad, quienes tienden a adoptar metas de rendimiento. Aunque en torno a esta idea, la hipótesis ha sido rebatida en diversas ocasiones, mostrando resultados poco concluyentes (e.g., Elliot y Church, 1997; Midgley y Urdan, 2001; Ross et al., 2002; Skaalvik, 1997).

Por otra parte, en relación al sexo, los resultados al respecto son bastante variados, pero complementarios entre sí. Tras la revisión de Long et al (2007), se observó que mientras que algunos estudios (e.g., Meece y Miller, 2001; Middleton y Midgley, 1997) únicamente reportaban que existían diferencias en las metas de evitación al rendimiento siendo los chicos los que eran más propensos a tener este tipo de orientación que las chicas, otros estudios (e.g., Anderman y Midgley, 1997; Nuñez et al., 1998; Pajares et al., 2000) únicamente proporcionaron evidencias de diferencias en las metas de aprendizaje, siendo las mujeres las que más propensas eran de manifestar este tipo de orientación motivacional. Asimismo, trabajos como el de Delgado et al. (2010), constataron que se daba una mezcla de ambas ideas: Por una parte, los chicos presentaron mayor puntuación en las metas de refuerzo social, mientras que las chicas presentaron mayor puntuación en las metas académicas y de logro. Cabe señalar que en todos los casos el tamaño del efecto fue pequeño.

Estos resultados servirían de base para apoyar la idea de que mientras que los chicos presentaban orientaciones motivacionales de origen más extrínsecos, las chicas parecen tener una orientación hacia el aprendizaje más intrínseco, relacionado con el interés y el esfuerzo de la tarea (De la Fuente, 2002).

Relacionado con esta idea, Cerezo y Casanova (2004) constataron que mientras los chicos manifestaban patrones atribucionales más externos ante el fracaso, las chicas tendían más a responsabilizarse en mayor medida de los malos resultados académicos, apuntan a una falta de esfuerzo o habilidad personal, siendo ambos factores internos. 
En el panorama adolescente español, una de las mayores aportaciones a la teoría de orientación a la meta es el estudio de García et al. (1998), quienes presentan un instrumento que permite incidir en conocer más cercanamente el patrón motivacional del alumnado y en base a los resultados, permitir reconducirlo hacia metas de aprendizaje (García et al., 1998). La estructura factorial desprendía tres factores: metas de aprendizaje, metas de valoración social y metas de recompensa.

Por una parte, tal y como se ha comentado anteriormente, las metas de aprendizaje proveen información sobre si los y las estudiantes están orientadas más hacia el proceso con el que llevan a cabo el aprendizaje o más hacia el resultado final (Valdez-García et al., 2017). Aquel alumnado con un patrón adaptativo persigue buscar el incremento de su propia competencia mediante la adquisición y dominio de nuevas habilidades y conocimientos (metas de aprendizaje), mientras que el que presenta un patrón motivacional desadaptativo está orientado hacia metas de ejecución o rendimiento, tratando de obtener exclusivamente un buen rendimiento, centrándose en el producto final y no en el proceso de aprendizaje (García et al., 1998).

Seguidamente, las metas de valoración social, a pesar de no guardar relación con el aprendizaje y el logro académico, son de especial importancia debido a que se basan en la experiencia emocional que viven los y las estudiantes ante las reacciones de personas significativas para estos (familia, docentes, iguales, etc.) ante su propia actuación. Lo óptimo para satisfacer este tipo de metas es conseguir un grado óptimo de aceptación social y evitar ser rechazado, como resultado de la conducta académica (González et al., 1996, p. 49).

Finalmente, las metas de recompensa hacen relación a la consecución de premios o recompensas, así como la evitación de todo lo que signifique castigo o pérdida de situaciones u objetos valorados por el sujeto en el proceso de aprendizaje (González et al 1996).

Las tres dimensiones estudiadas e investigadas por García et al (1998) y las dimensiones analizadas y más aceptadas en el entorno investigador no son excluyentes, sino que más bien se complementan. De este modo, por una parte, en ambos casos perciben la existencia de metas de aprendizaje y por otra parte, mientras el entorno investigador concibe la existencia de metas de aproximación al rendimiento y metas de evitación al rendimiento, García et al. (1998) las denomina a ambas metas de valoración social. En este sentido nos encontramos en el instrumento tanto ítems pertenecientes a las metas de aproximación al rendimiento (e.g., "Yo estudio porque quiero ser valorado por mis amigos" o "Yo estudio porque quiero que la gente vea lo inteligente que soy") como a las metas de evitación al rendimiento (e.g., "Yo estudio porque no quiero que mis compañeros se burlen de mí" o "Yo estudio porque no quiero que ningún profesor me tenga manía"). Finalmente, las metas de recompensa son entendidas como la motivación que presentan los alumnos y alumnas para aprender con el objetivo de conseguir un logro (e.g., "Yo estudio porque quiero obtener buenas notas").

La intensidad con la que se manifiesta cada una de estas metas académicas viene condicionada por una serie de variables tanto personales como sociales. De la Fuente (2004) destaca entre las más significativas la concepción que tengan los alumnos sobre qué es y cómo se desarrolla la inteligencia (Dweck, 1986; Nicholls, 1984; Valle et al., 1998), la personalidad (Covington, 2000; Harackiewicz et al., 2000) y/o la condición de riesgo social (Carroll et al., 1999).

Este campo de estudio, como son las metas académicas, ha cobrado especial interés en la literatura motivacional (Urdan, 2004). No obstante, es de destacar que gran parte de estos estudios muestran limitaciones de muestras poco representativas (Delgado et al., 2010) y la necesidad de investigación más profunda de diversas variables biológicas como el sexo y la edad (Cerezo y Casanova, 2004; Delgado et al., 2010; Meece, 2006; Pintrich y Schunk, 2002) en la etapa de educación primaria y en el ámbito español. Es por este motivo que a través del presente trabajo se tratará de conocer la correlación de las diferentes metas académicas y de conocer las diferencias de sexo y curso en estudiantes de educación primaria. 


\section{Método}

\section{Muestra}

La muestra completa del estudio estuvo conformada por 1059 estudiantes (539 chicas y 520 chicos), provenientes de un total de 18 centros educativos de diferentes comunidades autónomas de España: Cantabria, Comunidad Valenciana, Comunidad de Madrid, Aragón, País Vasco, Comunidad de Murcia y Canarias. 135 estudiantes eran de $4^{\mathrm{o}}, 310$ de $5^{\circ}$ y 614 de $6^{\circ}$ de Educación primaria $(\overline{\mathrm{x}}=10.45$; $\mathrm{DT}=.709)$.

Se decidió no dar oportunidad de participar a cursos inferiores a $4^{\circ}$ debido a que podría ponerse en riesgo la fiabilidad de los datos obtenidos, debido a que el instrumento original fue validado exclusivamente en alumnado del último ciclo de Educación Primaria, así como toda la Educación Secundaria Obligatoria.

\section{Instrumento}

Para medir la orientación motivacional de los y las estudiantes se empleó el cuestionario de metas académicas (García et al., 1998).

Este instrumento, compuesto por 20 ítems medidos en escala Tipo Likert de 5 puntos, indica que su estructura factorial está compuesto por tres dimensiones analizadas en el marco teórico de este estudio: Metas de aprendizaje, metas de valoración social y metas de recompensa.

\section{Procedimiento}

Tras obtener los permisos pertinentes de equipo directivo, docentes y familias de cada estudiante en cada centro para poder realizar el presente trabajo, se obtuvieron los datos de los participantes vía online. Para realizar todos los análisis se ha empleado el paquete estadístico SPSS (versión 24).

\section{Resultados}

\section{Análisis preliminares}

Antes de comenzar a dar respuesta a los interrogantes que plantea el estudio se comenzó eliminando los casos atípicos para las tres dimensiones estudiadas.

De seguido, se realizó un AFE para comprobar si los ítems originales a la escala se agrupaban en los tres factores iniciales planteados. La medida de adecuación muestral de KMO (.875) y la prueba de esfericidad d Bartlett $(\chi 2=7645.42 ; \mathrm{gl}=190 ; p=.000)$ indicaron la posibilidad de efectuar este análisis.

El AFE se efectuó a través del método de componentes principales, utilizando como criterio de selección el criterio de la raíz latente (autovalores mayores a 1) y empleando el método Varimax como método de rotación de la solución.

Los resultados a este AFE, mostrados en la Tabla 1, confirman la estructura factorial de tres dimensiones del cuestionario original. Estas tres dimensiones explican el $52.45 \%$ de la varianza; casi la misma que el cuestionario original con un $56.6 \%$ (García et al., 1998).

Posteriormente, se comprobaron los requisitos para pruebas paramétricas de normalidad, a través del estudio de la curtosis y la asimetría, y la homogeneidad de varianza, a través del estadístico de Levene. Los resultados mostraron que las variables poseían una distribución normal al tener todos los valores de asimetría y curtosis comprendidos entre \pm 2 (George y Mallery, 2010; Field, 2009; Gravetter y Wallnau, 2014; Trochim y Donnelly, 2006). En referencia al test de Levene, todos los análisis tuvieron significancia superior a .05 , por lo que asumimos que existe homogeneidad de varianza.

Finalmente, se estudió la consistencia interna del instrumento empleado, obteniendo resultados favorables. El valor general de la escala fue de $\alpha=.812$, mientras que los valores para las dimensiones de metas académicas, metas 
Tabla 1.

Estructura factorial rotada del cuestionario

\begin{tabular}{|c|c|c|c|c|}
\hline Ítems & & F1 & F2 & F3 \\
\hline Yo estudio porque para mí es interesante resolver problemas/tareas. & & .763 & & \\
\hline Yo estudio porque me gusta ver cómo voy avanzando & & .706 & & \\
\hline Yo estudio porque me gusta conocer nuevas cosas & & .755 & & \\
\hline $\begin{array}{l}\text { Yo estudio porque me gusta superar la dificultad que plantean los problemas/tareas } \\
\text { difíciles }\end{array}$ & & .706 & & \\
\hline Yo estudio porque me siento bien cuando supero obstáculos y/o fracaso & & .558 & & \\
\hline Yo estudio porque me gusta aprender cosas nuevas & & .768 & & \\
\hline Yo estudio porque me gusta utilizar la cabeza (mis conocimientos) & & .696 & & \\
\hline Yo estudio porque me siento muy bien cuando resuelvo problemas/tareas difíciles & & .643 & & \\
\hline Yo estudio porque quiero ser alabado por y padres y profesores & & & .685 & \\
\hline Yo estudio porque quiero ser valorado por mis amigos & & & .759 & \\
\hline Yo estudio porque no quiero que mis compañeros se burlen de mí & & & .725 & \\
\hline Yo estudio porque no quiero que ningún profesor me tenga manía & & & .717 & \\
\hline Yo estudio porque quiero que la gente vea lo inteligente que soy & & & .769 & \\
\hline Yo estudio porque deseo obtener mejores notas que mis compañeros & & & .748 & \\
\hline Yo estudio porque quiero obtener buenas notas & & & & .733 \\
\hline Yo estudio porque quiero sentirme orgulloso de obtener buenas notas & & & & .772 \\
\hline Yo estudio porque no quiero fracasar en los exámenes finales & & & .313 & .608 \\
\hline Yo estudio porque quiero terminar bien mis estudios & & & & .733 \\
\hline Yo estudio porque quiero conseguir un buen trabajo en el futuro & & & & .637 \\
\hline \multirow[t]{3}{*}{ Yo estudio porque quiero ser una persona importante en el futuro } & & & .426 & .344 \\
\hline & $\lambda$ & $26.01 \%$ & $17.16 \%$ & $9.28 \%$ \\
\hline & $\lambda_{T}$ & & $52.45 \%$ & \\
\hline
\end{tabular}

Nota: Factor 1: Metas académicas; Factor 2: Metas de valoración social; Factor 3: Metas de recompensa.

de valoración social y metas de recompensa fueron de $\alpha=.859, \alpha=.838$ y $\alpha=.735$, respectivamente.

\section{Análisis de coorrelaciones}

Esta subsección presenta el análisis de los resultados vinculados a la correlación existente entre las diferentes dimensiones del cuestionario de metas académicas. To- 
mando como base este análisis, se pretende conocer si las correlaciones entre estas dimensiones son significativas, hecho que apoyaría la idea de que la orientación motivacional hacia el aprendizaje del alumnado de educación primaria sería multifactorial y no única.

Para este propósito se realizó un análisis correlacional a través del estadístico $r$ de Pearson, ilustrado en la Tabla 2.

Tabla 2.

Correlaciones entre las dimensiones estudiadas

\begin{tabular}{cccc}
\hline & MA & MVS & MR \\
\hline MA & 1 & $.093^{* *}$ & $.344^{* * *}$ \\
MVS & - & 1 & $.367^{* * *}$ \\
MR & - & - & 1 \\
\hline
\end{tabular}

Nota: ${ }^{*}<.05 ;{ }^{* *}<.01 ;{ }^{* * *}=.00$. MA: Metas académicas; MVS: Metas de valoración social; ML: Metas de recompensa; $\mathrm{n}=1059$.

De estos resultados, se observa que todos los tipos de metas correlacionan positiva y significativamente entre sí. Se destaca la correlación de intensidad entre débil y moderada de las metas académicas con las metas de recompensa $(r=.344 ; p=.000) \mathrm{y}$ de las metas de valoración social con las metas de recompensa $(r=.367 ; p=.000)$, en contraposición a los valores estadísticamente significativos, pero de intensidad de correlación muy baja de las metas académicas con las metas de valoración social $(r=.093 ; p=.003)$

Tabla 3.

Prueba $T$ entre sexos

\section{Diferencias de sexo}

Posteriormente, en esta subsección se presentan los resultados obtenidos de realizar un análisis de comparación de medias entre sexos.

Este análisis ayudaría a conocer si los estudiantes chicos presentan diferente tipo de orientación motivacional a sus coetáneos chicas.

Tal y como queda recogido en la Tabla 3 , se puede apreciar que, respecto a las metas de recompensa, ambos sexos poseen valores muy similares y estadísticamente no significativos. Sin embargo, se han encontrado diferencias estadísticamente significativas en las metas académicas y en las metas de valoración social.

En referencia a las metas académicas, observamos que las chicas $(\overline{\mathrm{x}}=4.118$; $\mathrm{DT}=.680)$ obtuvieron valores superiores a sus coetáneos chicos $(\overline{\mathrm{x}}=4.032 ; \mathrm{DT}=.683)$. Estas diferencias entre ambos grupos fueron, sin embargo, bastante pequeñas ( $d=-.12$; Cohen, 1988). Por otra parte, en las metas de valoración social, ocurrió al contrario que en el caso anterior: 10 chicos $(\overline{\mathrm{x}}=2.949$; $\mathrm{DT}=1.117)$ obtuvieron valores considerablemente superiores a las chicas $(\bar{x}=2.598 ; \mathrm{DT}=1.095)$, siendo el tamaño del efecto de estas diferencias entre pequeñas y medianas $(d=.31$; Cohen, 1988).

\section{Diferencias por curso}

En último lugar, se analizaron las diferencias existentes entre los cursos estudiados en educación primaria a través de una ANOVA de un factor.

\begin{tabular}{|c|c|c|c|c|c|c|c|}
\hline & \multicolumn{2}{|c|}{ Chicos } & \multicolumn{2}{|c|}{ Chicas } & \multirow{2}{*}{$t$} & \multirow{2}{*}{ Sig. } & \multirow{2}{*}{$d$} \\
\hline & $\overline{\mathbf{x}}$ & $D T$ & $\overline{\mathbf{x}}$ & $D T$ & & & \\
\hline MA & 4.032 & .683 & 4.118 & .680 & -2.047 & .041 & -.12 \\
\hline MVS & 2.949 & 1.117 & 2.598 & 1.095 & 5.151 & .000 & .31 \\
\hline ML & 4.544 & .525 & 4.520 & .569 & .717 & .473 & - \\
\hline
\end{tabular}

Nota: MA, Metas académicas; MVS, Metas de valoración social; ML, Metas de recompensa. $n=1059$. El valor d de Cohen negativo implica un tamaño del efecto favorable del grupo 2 (Femenino) sobre el grupo 1 (Masculino). 
Tabla 4.

ANOVA entre los cursos estudiados

\begin{tabular}{cccccccccc}
\hline & \multicolumn{2}{c}{$\mathbf{4}^{\mathbf{0}}$} & \multicolumn{2}{c}{$\mathbf{5}^{\mathbf{0}}$} & \multicolumn{2}{c}{$\mathbf{6}^{\mathbf{0}}$} & Sig. & Post-Hoc & $\boldsymbol{\eta}^{2}$ \\
\cline { 2 - 8 } & $\overline{\mathbf{x}}$ & $\boldsymbol{D T}$ & $\overline{\mathbf{x}}$ & $\boldsymbol{D T}$ & $\overline{\mathbf{x}}$ & $\boldsymbol{D T}$ & & & \\
\hline MA & 4.190 & .569 & 4.137 & .668 & 4.020 & .707 & .005 & $(1-3)(2-3)$ & .010 \\
MVS & 3.426 & 1.089 & 2.871 & 1.087 & 2.578 & 1.082 & .000 & $(1-2)(1-3)(2-3)$ & .063 \\
MR & 4.539 & .507 & 4.519 & .563 & 4.537 & .549 & .885 & - & - \\
\hline
\end{tabular}

Nota: MA, Metas académicas; MVS, Metas de valoración social; ML, Metas de recompensa. $n=1059$. Post-Hoc: Scheffe. $4^{\circ}$ de Educación Primaria (1); $5^{\circ}$ de Educación Primaria (2); $6^{\circ}$ de Educación Primaria (3).

Este análisis, ayudaría a conocer si realmente la orientación motivacional se mantiene estable con el paso de los cursos, o si, por el contrario, a medida que el curso avanza, y por ende, la edad, la orientación para aprender de los y las estudiantes de educación primaria también varía.

Los resultados, mostrados en la Tabla 4, apuntan a que mientras no se hallaron diferencias significativas en las metas de recompensa $(p=.885)$, sí que se hallaron diferencias significativas en la orientación motivacional de las metas académicas y las metas de valoración social con el paso de los cursos.

Más concretamente, se aprecia como con el paso de los cursos, la orientación motivacional hacia metas de aprendizaje tiende a disminuir lentamente, pero significativamente $\left(p=.005 ; \eta^{2}=.010\right)$ al igual que ocurre con las metas de valoración social, que también disminuyen, aunque estas con una intensidad más fuerte que las anteriores $\left(p=.000 ; \eta^{2}=.063\right)$.

\section{Discusión}

El presente estudio tuvo como objetivo conocer la relación entre los diferentes tipos de metas académicas que el alumnado de educación persiguen, además de conocer las diferencias existentes por sexo y curso. El propósito de este trabajo era investigar si los hallazgos encontrados en otros estudios con muestras dispares, alejadas de la educación básica podían ser igualmente de aplicables al tipo de muestra con el que se ha realizado el trabajo. De este modo, la teoría de orientación a la meta podría ser utili- zada como referente en aras de estudiar la motivación académica de los y las estudiantes.

Los hallazgos obtenidos en el presente trabajo sobre la correlación entre las diversas metas académicas apuntan a que existe una correlación positiva entre las tres diferentes metas estudiadas. De igual modo, cabe recalcar que la fuerza de correlación entre las diferentes metas oscila entre baja y moderada, resultados análogos a otros trabajos como los de Middleton y Midgley (1997). Los resultados obtenidos indican que la orientación motivacional hacia el aprendizaje no se da exclusivamente por la presencia de una única meta y eliminando el resto, sino que, por el contrario, varía la intensidad con la que se manifiesta cada una de ellas. Esta idea es soportada por otra serie de estudios como el de Heyman y Dweck (1992), Meece (1994), Ross et al. (2002) o Seifert (1995), entre otros.

Referente al sexo, los resultados obtenidos sustentan la idea de que mientras los chicos tienden más a presentar una orientación motivacional más centrada en la valoración social, las chicas tienden a presentar puntuaciones mayores en perfiles orientacionales más relacionados con las metas académicas.

Estos resultados van acorde a las conclusiones sacadas en otro tipo de muestras que sustentan la idea de que las chicas se muestran más favorables por desarrollar su capacidad y destacar en el rendimiento académico, mientras que los chicos se centran más la realización actividades que les otorguen prestigio social (Sanz de Acedo et al., 2003). En palabras de Castillo et al. (2003), este hecho podría estar ocurriendo debido a que los chicos aprenden a compararse con los demás para validar su competencia con la intención de ganar a toda costa, mientras que las 
chicas suelen compararse con ellas mismas con la intención de realizar la tarea de manera más óptima. Cabe destacar en el caso de los chicos, que un tipo de perfil motivacional basado en la obtención de juicios de aprobación y evitación del rechazo familiar y docente puede generar, indirectamente, una mayor probabilidad de alcanzar un bajo rendimiento académico (Covington, 2000), por lo que este hecho debería tenerse en cuenta en la praxis educativa en el momento de desarrollar estrategias de intervención que traten de rebajar este patrón motivacional desadaptativo (Martín, 2004).

Por otra parte, sobre el curso escolar, los datos obtenidos nos muestran como no solo hay diferencias a nivel de sexo, ya que, a medida de aumentar el curso escolar, las metas de carácter académico van disminuyendo lenta y progresivamente, mientras que las metas de valoración social disminuyen de manera intensa a medida que aumenta la edad del alumnado.

Esta tesis ha sido contrastada por otros estudios, como el de Wigfield y Wagner (2005), con el que se constató que en la transición de primaria a secundaria ocurre un descenso importante de la motivación hacia el aprendizaje y el estudio. Asimismo, la literatura científica de diferentes países como Canadá (Bouffard et al., 1998), China (Fung et al., 2007) o España (Sanz de Acedo et al., 2003) sostiene la idea de que las metas de aprendizaje suelen ser más favorables en educación primaria y progresivamente van disminuyendo hasta llegar a los últimos cursos de la etapa de educación secundaria, siendo realmente impactante el cambio de primaria a secundaria. Este fenómeno podría estar originado por el aumento de las dificultades de las tareas escolares y por la disminución de la percepción de competencia del estudiante. Además, la necesidad de un refuerzo positivo por parte de la familia o el docente, tan característica en educación infantil, disminuye notablemente durante la adolescencia, ya que en esta etapa la cognición madura notablemente (Delgado et al., 2010).

Se cree que, en vista de todos estos resultados, futuros trabajos podrían centrarse no solo en conocer más el patrón motivacional de estudiantes de educación primaria, sino también en conocer cómo varía el patrón motivacional en momentos críticos de la vida del alumnado, como el cambio de educación primaria, el cambio de educación secundaria bachillerato o ciclos formativos, el cambio de bachillerato a la universidad, etc.

De igual modo, podría resultar interesante para la comunidad científica realizar investigación más profunda en dar a conocer qué perfil docente se relaciona más favorablemente con cada una de las diferentes metas académicas en diferentes etapas educativas.

\section{Referencias}

Ames, C. (1992). Classrooms: Goals, Structures, and Student Motivation. Journal of Educational Psychology, 80, 260-267.

Anderman, E. M., y Midgley, C. (1997). Changes in Achievement Goal Orientations, Perceived Academic Competence, and Grades across the Transition to Middle-Level Schools. Contemporary Educational Psychology, 22, 269-298.

Bouffard, T., Vezeau, C. y Bordeleau, L. (1998). A Developmental Study of the Relation between Combined Learning and Performance Goals and Students' Self-Regulated Learning. British Journal of Educational Psychology, 68(3), 309-319. https://doi.org/10.1111/j.2044$\underline{\text { 8279.1998.tb01293.x }}$

Brophy, J. (2005). Goal Theorists Should Move on from Performance Goals. Educational Psychologist, 40(3), 167-176. https://doi.org/10.1207/s15326985ep4003_3

Carroll, A., Baglioni, J. R., Houghton, S. y Bramston, P. (1999). At-risk and not At-Risk Primary School Children: An Examination of Goal Orientations and Social Reputations. British Journal of Educational Psychology, 69, 377-392. https://doi.org/10.1348/000709999157789

Castillo, I., Balaguer, I. y Duda, J. (2003). Las teorías personales sobre el logro académico y su relación con la alienación escolar [Personal Theories of 
Academic Achievement and their Relationship to Alienation from School]. Psicothema, 15(1), 7581.

Cerezo, M. T. y Casanova, P. F. (2004). Diferencias de género en la motivación académica de los alumnos de Educación Secundaria Obligatoria. Revista Electrónica de Investigación Psicoeducativa, 2(1), 97-112.

Cohen, J. (1988). Statistical Power Analysis for the Behavioral Sciences (2 ${ }^{\mathrm{a}}$ ed.). Lawrence Earlbaum.

Covington, M. V. (2000). Goal Theory, Motivation and School Achievement: An Integrative Review. Annual Review of Psychology, 51, 171-200. https://doi.org/10.1146/annurev.psych.51.1.171

De la Fuente, J. (2004). Perspectivas recientes en el estudio de la motivación: La teoría de la Orientación de Meta [Recent Perspectives on the Study of Motivation: The Goal Orientation Theory]. Escritos de Psicología, 6, 72-84.

Delgado, B., Inglés, C. J., García-Fernández, J. M., Castejón J. L. y Valle, A. (2010). Diferencias de género y curso en metas académicas en alumnos de Educación Secundaria Obligatoria. Revista Española de Pedagogía, 245, 67-84.

Dweck, C. S. (1986). Motivational Processes Affecting Learning. American Psychologist, 41, 1040-1048. https://doi.org/10.1037/0003-066X.41.10.1040

Dweck, C. S. y Legget, E. L. (1988). A Social-Cognitive Approach to Motivation and Personality. Psychological Review, 95, 256-273. https://doi.org/10.1037/0033-295X.95.2.256

Elliot, A. J. y Church, M. A. (1997). A Hierarchical Model of Approach and Avoidance Achievement Motivation. Journal of Personality and Social Psychology, 72, 218-232. https://doi.org/10.1007/s11031-006-9028-7
Elliot, A. J. y Harackiewicz, J. M. (1996). Approach and Avoidance Achievement Goals and Intrinsic Motivation: A Mediational Analysis. Journal of Personality and Social Psychology, 70, 461-475. https://doi.org/10.1037/0022-3514.70.3.461

Field, A. (2009). Discovering Statistics using SPSS. SAGE.

Fung, Y., Mei-Tai, R. y Sun-Keung, N. (2007). Developmental Patterns of School Students' Motivational and Cognitive-Metacognitive Competences. Educational Studies, 33 (1), 81-98. https://doi.org/10.1080/03055690600948281

García, M., González-Pienda, J., Núñez, J. C., GonzálezPumariega, S., Álvarez, L., Roces, C., González, R. y Valle, A. (1998). El cuestionario de metas académicas (C.M.A.). Un instrumento para la evaluación de la orientación motivacional de los alumnos de educación secundaria. Aula Abierta, 71, 175-199.

George, D. y Mallery, M. (2010). SPSS for Windows Step by Step: A Simple Guide and Reference, 17.0 update. Pearson.

González, R. Valle, A., Núñez, J. C. y González-Pienda, J. A. (1996). Una aproximación teórica al concepto de metas académicas y su relación con la motivación escolar [A Theoretical Approach to Concept of Academic Goals and their Relationship Withschool Motivation]. Psicothema, 8(1), 45-61.

Gravetter, F. y Wallnau, L. (2014). Essentials of Statistics for the Behavioral Sciences. Wadsworth.

Harackiewicz, J. M., Barron, K. E. y Elliot, A. J. (1998). Rethinking Achievement Goals: When are they Adaptive for College Students and why? Educational Psychologist, 33, 1-21. https://doi.org/10.1207/s15326985ep3301 1

Harackiewicz, J. M., Barron, K. E., Tauer, J. M., Carter, S. M. y Elliot, A. J. (2000). Short-term and LongTerm Consequences of Achievement Goals: 
Predicting Interest and Performance Over Time. Journal of Educational Psychology, 92(2), 316330. https://doi.org/10.1037//0022-0663.92.2.316

Heyman, G. y Dweck, C. S. (1992). Achievement Goals and Intrinsic Motivation: Their Relation and their Role in Adaptive Motivation. Motivation and Emotion, 16(3), 231-247.

Kohler, J. y Reyes, M. (2010). Teoría de orientación a la meta: Hallazgos y aplicaciones en la educación y la educación física [Theory of Goal-Oriented: Findings and Applications in Education and Physical Education]. Revista Cultura, 24, 243-265.

Linnenbrink, E. y Pintrich, P. R. (2000). Multiple Pathways to Learning and Achievement: The Role of Goal Orientation in Fostering Adaptive Motivation, Affect, and Cognition. En C. Sansone y J. M. Harackiewicz (Eds.), Intrinsic and Extrinsic Motivation: The Search for Optimal Motivation and Performance (pp. 195-227). Academic Press.

Long, J., Monoi, S., Harper, B., Knoblauch, D. \& Murphy, K. (2007). Academic Motivation and Achievement Among Urban Adolescents. Urban Education, 42, 196-122. https://doi.org/10.1177/0042085907300447

Maehr, M. L. y Midgley, C. (1996). Transforming School Cultures. Westview Press.

Martin, A. J. (2004). School Motivation of Boys and Girls: Differences of degree, Differences of Kind, or both? Australian Journal of Psychology, 56(3), 133-146. https://doi.org/10.1080/00049530412331283363

Matos, L. y Lens, W. (2006). La teoría de Orientación a la Meta, estrategias de aprendizaje y rendimiento académico en estudiantes de secundaria de Lima [The Goal Orientation Theory, Learning Strategies and Academic Achievement in Secondary Students in Lima]. Persona, 9, 11-30.
Meece, J. L. (1994). The Role of Motivation in SelfRegulated Learning. En D. H. Schunk y B. J. Zimmerman (Eds.), Self-Regulation of Learning and Performance: Issues and Educational Applications (pp. 25-44). Lawrence Erlbaum.

Meece, J. L, Bower, B y Burg, S. (2006). Gender and Motivation. Journal of School Psychology, 44, 351-373. https://doi.org/10.1016/J.JSP.2006.04.004

Meece, J. L. y Miller, S. D. (2001). A Longitudinal Analysis of Elementary School Students' Achievement Goals in Literacy Activities. Contemporary Educational Psychology, 26, 454480. https://doi.org/10.1006/ceps.2000.1071

Middleton, M. J., y Midgley, C. (1997). Avoiding the Demonstration of Lack of Ability: An Underexplored Aspect of Goal Theory. Journal of Educational Psychology, 89(4), 710-718. https://doi.org/10.1037/0022-0663.89.4.710

Midgley, C. y Urdan, T. (2001). Academic SelfHandicapping and Achievement Goals: A Further Examination. Contemporary Educational Psychology, 26, 61-75. https://doi.org/10.1006/ceps.2000.1041

Miller, R. B., Behrens, J. T., Greene, B. A. y Newman, D. (1993). Goals and Perceived Ability: Impact on Student Valuing, Self-Regulation, and Persistence. Contemporary Educational Psychology, 18(1), 214. https://doi.org/10.1006/ceps.1993.1002

Nicholls, J. G. (1984). Achievement Motivation: Conceptions of Ability, Subjective Experience, Task Choice and Performance. Psychological Review, 91, 328-346. https://doi.org/10.1037/0033-295X.91.3.328

Nuñez, J. C., González-Pienda, J. A., García, M. S., González-Pumariega, S. y García, S. I. (1998). Estrategias de aprendizaje en estudiantes de 10 a 14 años y su relación con los procesos de atribución 
causal, el autoconcepto y las metas de estudio. Estudios de Psicología, 19(59), 65-85.

Pajares, F., Britner, S. L. y Valiante, G. (2000). Writing and Science Achievement Goals of Middle School Students. Contemporary Educational Psychology, 25, Artículo 4067422.

Patrick, H., Anderman, L. H., Ryan, A. M., Edelin, K. C. y Midgley, C. (2001). Teachers' Communication of Goal Orientations in Four Fifth-Grade Classrooms. The Elementary School Journal, 102(1), 35-58. https://doi.org/10.1086/499692

Pintrich, P. R. (2000). Multiple Goals, Multiple Pathways: The Role of Goal Orientation in Learning and Achievement. Journal of Educational Psychology, 92, 544-555.

Pintrich, P. R. y Schunk, D. H. (2002). Motivation in Education: Theory, Research, and Applications. Prentice-Hall.

Pintrich, P. R. y De Groot, E. V. (1990). Motivational and Self-Regulated Learning Components of Classroom Academic Performance. Journal of Educational Psychology, 82, 33-40.

Ross, M., Shannon, D., Salisbury-Glennon, J. y Guarino, A. (2002). The Patterns of Adaptive Learning Survey: A Comparison across Grade Levels. Educational and Psychological Measurement, 62, 483-497.

https://doi.org/10.1177/00164402062003006

Ross, M., Blackburn, M. y Forbes, S. (2005). Reliability Generalization of the Patterns of Adaptive Learning Survey Goal Orientation Scales. Educational and Psychological Measurement, 65, 451-464. https://doi.org/10.1177/0013164404272496

Sanz-de-Acedo, M. L., Ugarte, M. D. y Lombreras, M. V. (2003). Desarrollo y validación de un Cuestionario de Metas para Adolescentes [Development and Validation of an Adolescents
Goals Questionnaire.]. Psicothema, 15(3), 493499.

Seifert, T. L. (1995). Academic Goals and Emotions: A Test of Two Models. The Journal of Psychology: Interdisciplinary and Applied, 129(5), 543-552. https://doi.org/10.1080/00223980.1995.9914926

Skaalvik, E. M. (1997). Self-enhancing and SelfDefeating Ego Orientation: Relations with Task and Avoidance Orientation, Achievement, SelfPerceptions and Anxiety. Journal of Educational Psychology, $\quad$ 89(1), 71-81. https://doi.org/10.1037/0022-0663.89.1.71

Trochim, W. M. y Donnelly, J. P. (2006). The Research Methods Knowledge Base. Atomic Dog.

Urdan, T. (2004). Predictors of Academic SelfHandicapping and Achievement: Examining Achievement Goals, Classroom Goal Structures and Culture. Journal of Educational Psychology, 96, 251-264. https://doi.org/10.1037/0022$\underline{0663.96 .2 .251}$

Valdez-García, J. E., López, M. V. y Olivares, S. L. (2017). Definición de metas de aprendizaje en estudiantes de pregrado en un curso de Historia de la medicina [Definition of Educational Objectives of Medical Students in a History of Medicine Class]. Educación Médica, 19(2), 105-110. https://doi.org/10.1016/j.edumed.2017.03.027

Valle, A., González, R., Gómez, M. L., Rodríguez, S. y Piñeiro, I. (1998). Influencia de las atribuciones causales internas y externas sobre las metas académicas. Bordón, 50(4), 405-413.

Wingfield, A. y Wagner, A. L. (2005). Competence, Motivation and Identity Development during Adolescence. En C. Dweck y A. Elliot (Eds), Handbook of Competence and Motivation (pp. 222-239). Guilford. 